\title{
Adiabatic analysis of the rotating BTZ black hole
}

\author{
Mohsen Fathi ${ }^{1, \mathrm{a}}$, Samuel Lepe ${ }^{2, \mathrm{~b}}$, J. R. Villanueva ${ }^{1, \mathrm{c}}$ \\ ${ }^{1}$ Instituto de Física y Astronomía, Universidad de Valparaíso, Avenida Gran Bretaña 1111, Valparaíso, Chile \\ ${ }^{2}$ Instituto de Física, Pontificia Universidad de Católica de Valparaíso, Avenida Brasil 2950, Valparaíso, Chile
}

Received: 8 March 2021 / Accepted: 29 May 2021 / Published online: 6 June 2021

(C) The Author(s) 2021

\begin{abstract}
In this paper we analyze some interesting features of the thermodynamics of the rotating BTZ black hole from the Carathéodory axiomatic postulate, for which, we exploit the appropriate Pfaffian form. The allowed adiabatic transformations are then obtained by solving the corresponding Cauchy problem, and are studied accordingly. Furthermore, we discuss the implications of our approach, regarding the second and third laws of black hole thermodynamics. In particular, the merging of two extremal black holes is studied in detail.
\end{abstract}

\section{Contents}

1 Introduction ................. 1

2 The rotating BTZ black hole and its thermodynamics 2

3 The adiabatic-isoperimetral transformations . . . . . 4

4 Scattering of two extremal black holes and the second law .................. . . 6

5 Discussion and final remarks . . . . . . . . . . 7

References ............... 7

\section{Introduction}

The $(2+1)$-dimensional topological gravity has appeared to be of interest ever since the late twentieth century, especially because it can be used as a toy model for quantum gravity; the property that was discovered after the arguments presented regarding the possible connections between the (2+1)-dimensional gravity and the Chern-Simons theory $[1,2]$. In the same realm, the Chilean physicists, Bañados, Teitelboim, and Zanelli (BTZ), proposed a black hole solution in the $\mathrm{SO}(2,2)$ gauge group with a negative cosmological constant, which resembled, remarkably, the prop-

\footnotetext{
a e-mail: mohsen.fathi@postgrado.uv.cl

b e-mail: samuel.lepe@ pucv.cl

${ }^{\mathrm{c}}$ e-mail: jose.villanueva@uv.cl (corresponding author)
}

erties of the $(3+1)$-dimensional Schwarzschild and Kerr black holes [3], and hence had a convincing physical significance. This solution was given further refinements, corrections and generalizations [4-8], and later Witten calculated the entropy of the BTZ black holes [9]. This solution, in fact, has gone through numerous investigations, from different perspectives. For instance, one addressed the geodesic structure of the uncharged BTZ black holes [10], the scattering process of test particles [11,12], the quasi-normal modes [13-16], the hydrostatic equilibrium conditions for finite distributions [17], and solutions for fluid distributions matching the exterior BTZ spacetime [18-21]. Also, by considering a non-constant coupling parameter with the energy-scale, the scale-dependent version of the BTZ solution has been developed and discussed in Refs. [22-26]. It has been also argued that, if the energy-momentum complexes of Landau-Lifshitz and Weinberg are employed for a rotating BTZ black hole, the same energy distribution is obtained from both prescriptions [27]. This spacetime has been also generalized regarding the inclusion of terms related to the non-linear electrodynamics $[28,29]$ and the conformal group [30]. Furthermore, regarding the black hole thermodynamics, BTZ black holes have been investigated in terms of their critical behavior and phase transitions, by evaluating their equilibrium thermodynamic fluctuations in Ref. [31], where it is shown that the extremal BTZ black hole with angular momentum serves as the critical point, and the density of states in the micro- and grand-canonical ensembles has been calculated in Ref. [32]. For the case that the cosmological constant is considered as a thermodynamic parameter, Kerr-(Anti-)de Sitter and the BTZ black holes have been compared [33]. Furthermore, the quantum corrections to the enthalpy and the equation of state of the uncharged BTZ black holes were studied in Ref. [34]. In Ref. [35], a general class of BTZ black holes is studied regarding the Ruppeiner geometry of the thermodynamic state space, and it is found that this geometry is flat for both the rotating BTZ and the BTZ-Chern-Simons black holes, in the canonical ensemble. However, a non-zero scalar cur- 
vature is introduced to the thermodynamic geometry, when thermal fluctuations are included. In fact, this establishment of geometrothermodynamics, as well as that introduced in Ref. [36], is a formalism that is used to designate a flat twodimensional space of equilibrium states, which is endowed with a thermodynamic metric. This way, the space allows for the thermodynamic interaction, free of any kind of singularities (phase transitions). A more generalized consideration of geometrothermodynamics is available in Ref. [37], where the thermodynamics of the charged BTZ black hole is investigated in the context of the Weinhold and Ruppeiner geometries. There, it is shown that these geometries cannot describe completely the black hole thermodynamics and the corresponding criteria for the electric charge. To solve this problem, in Ref. [38], a new metric (the HPEM metric) was introduced through a specific formalism, and this way, the corresponding Ricci scalar was shown to be able to bring together different types of phase transitions. In Ref. [39], it is proved that the HPEM metric gives a consistent picture in the study of the thermodynamics of BTZ black holes. The inclusion of quantum scalar fields in the study of black hole thermodynamics was done thoroughly in Ref. [40], for the case of a static BTZ black hole, which leads to the introduction of entanglement thermodynamics for mass-less scalar fields. It has been also shown that the thermodynamics of BTZ black holes can be deformed in the context of gravity's rainbow; however, the Gibbs free energy remains unchanged [41]. Gravity's rainbow has been also exploited to study the black hole heat capacity and phase transition of BTZ black holes in Refs. [42,43]. There are also some other extensions of the BTZ black holes that are derived in alternative theories of gravity. For example, in Ref. [44], the Noether symmetries of the rotating BTZ black hole in $f(R)$ gravity have been used to generate new BTZ-type solutions. Along the same lines, in Ref. [45], some thermodynamic aspects of the BTZ black holes, such as the Carnot heat engine, are studied in the context of massive gravity. Also, the authors in Ref. [46] consider the Horndeski action as the source field of the BTZ black hole, and reduce it to the common Einstein-Hilbert action including a cosmological constant. This way, they regain the usual three-dimensional Smarr formula by exploiting the scaling symmetry of this reduced action. Moreover, rotating BTZ black holes have been shown to exhibit no kind of superradiance, if the considered Dirac fields vanish at infinity [47].

There is also an interesting issue, regrading the $(2+1)$ dimensional exotic black hole solutions, whose spacetime metric has the same form as that of the BTZ, however, with mass and angular momentum being reversed in their roles [48]. For this particular case, the entropy is proportional to the length of the inner horizon. The inner and outer horizons, in fact, limit the propagation of radial geodesics. Therefore, they can be thermally quantized only beyond the horizons. Accordingly, it has been shown in Ref. [49] that the entropy of the BTZ black holes is in agreement with the Bekenstein-Hawking formula, and the particles retain their quantum ground level in the BTZ spacetime.

The reason of presenting such a relatively long introduction is to highlight the interest of the scientific community in scrutinizing the thermodynamic of $(2+1)$-dimensional, and in particular, the BTZ black holes. In this paper, based on the same interest, we study some thermodynamic aspects of the rotating BTZ black hole. Specifically, we base our discussion on the Carathéodory postulate of adiabatic inaccessibility [50], which ensures the integrability of the Pfaffian form, $\delta Q_{\text {rev }}$, which represents the infinitesimal heat exchanged reversibly. This approach allows for constructing a proper thermodynamic manifold by means of foliating the adiabatic surfaces that satisfy the Pfaffian equation $\delta Q_{\text {rev }}=0$. For the case of the $(3+1)$-dimensional black holes, this type of construction has been studied in detail in Refs. [51-53], which gives rise to the isoareal transformations, i.e., transformations between the black hole states with the same areas. On the other hand, for $(2+1)$-dimensional black holes, the adiabatic transformations correspond to the isoperimetral transformations between states that reside in the non-extremal manifold.

In order to elaborate on this, in Sect. 2, we introduce the rotating BTZ solution, and the way we approach it is based on the Carathéodory postulate. In Sect. 3, we study the allowed adiabatic transformation for the thermodynamic states, given the corresponding analytical solutions that constitute an adiabatic hypersurface. The second law of black hole thermodynamics is then considered in more detail in Sect. 4, where we consider the scattering of two extremal BTZ black holes. We summarize the results in Sect. 5.

\section{The rotating BTZ black hole and its thermodynamics}

The (2+1)-dimensional, uncharged, black hole solution with a negative cosmological constant $\Lambda=-\ell^{-2}$ is obtained from the action

$I=\frac{c}{2 \pi G} \int \sqrt{-g}\left[R+2 \ell^{-2}\right] \mathrm{d}^{2} x \mathrm{~d} t+\mathcal{B}$,

where $\mathcal{B}$ is a surface term $[3,4]$. For the stationary circular symmetry, the corresponding spacetime metric is given in terms of the coordinates $-\infty<t<\infty, 0<r<\infty$, and $0 \leq \phi \leq 2 \pi$, and can be written as

$\mathrm{d} s^{2}=-N^{2}(r) c^{2} \mathrm{~d} t^{2}+N^{-2}(r) \mathrm{d} r^{2}+r^{2}\left[N^{\phi}(r) c \mathrm{~d} t+\mathrm{d} \phi\right]^{2}$, 
in which the square lapse function and the angular shift are given, respectively, by

$$
\begin{aligned}
& N^{2}(r)=-\frac{G M}{c^{2}}+\frac{r^{2}}{\ell^{2}}+\frac{G^{2} J^{2}}{4 c^{6} r^{2}}, \\
& N^{\phi}(r)=-\frac{G J}{2 c^{3} r^{2}},
\end{aligned}
$$

where $M$ and $J$ indicate the mass and the angular momentum of the black hole. This spacetime possesses an inner $\left(r_{-}\right)$and an event $\left(r_{+}\right)$horizon, which are located at

$r_{ \pm}=\frac{c \tau_{ \pm}(\mathcal{M}, \mathcal{J})}{\sqrt{2}}$

where

$\tau_{ \pm}(\mathcal{M}, \mathcal{J})=\sqrt{\mathcal{M} \pm \sqrt{\mathcal{M}^{2}-\mathcal{J}^{2}}}$

and

$\mathcal{M} \equiv \frac{M}{m_{p} \Omega_{\mathrm{ext}}^{2}}$,

$\mathcal{J} \equiv \frac{t_{p} J}{\hbar \Omega_{\mathrm{ext}}}$.

Here the subscript " $p$ " refers to the Planck quantities in $(2+1)$ dimensions, ${ }^{1}$ and $\Omega_{\mathrm{ext}}=c / \ell$ is the angular velocity of the extremal black hole. Note that the physical dimension of $\mathcal{M}$ and $\mathcal{J}$ is $\left[\right.$ time $\left.^{2}\right]$, while the function $\tau_{ \pm}$has the dimension of [time]. Also, in the extremal case the relation $\mathcal{M}=\mathcal{J}$ is satisfied.

The Bekenstein-Hawking entropy formula, if applied to the BTZ black hole, gives the entropy proportional to the event horizon's perimeter $P_{\mathrm{bh}}=2 \pi r_{+}$instead of its area $A_{\mathrm{bh}}$, as expected on dimensional grounds. Therefore

$S=\frac{k_{B}}{4} \frac{P_{\mathrm{bh}}}{\ell_{p}}=\frac{k_{B}}{4}\left(\frac{2 \pi r_{+}}{c t_{p}}\right)=a \tau_{+}$,

where $S$ is the entropy, $k_{B}$ is the Boltzmann constant, and $a=(\pi / \sqrt{8})\left(k_{B} / t_{p}\right) \approx 1.1\left(k_{B} / t_{p}\right)$. Defining $\mathcal{S} \equiv S / a=$ $\tau_{+}$, and using Eqs. (5) and (7), we obtain a Christodouloutype mass formula, which relates the total mass (energy) $\mathcal{M}$ to the entropy and the angular momentum, in the following form:

$\mathcal{M}(\mathcal{S}, \mathcal{J})=\frac{1}{2} \mathcal{S}^{2}+\frac{1}{2} \frac{\mathcal{J}^{2}}{\mathcal{S}^{2}}$

\footnotetext{
${ }^{1}$ In (2+1)-dimensional gravity, the gravitational constant $G$ has the physical dimension of [length ${ }^{2} /\left(\right.$ mass $\times$ time $\left.\left.^{2}\right)\right]$ (see Ref. [54]). Therefore, the Planck mass, length and time become $m_{p}=c^{2} / G, l_{p}=$ $G \hbar / c^{3}$, and $t_{p}=l_{p} / c$.
}

We base our study on the framework of Carathéodory's approach to thermodynamics, which postulates the integrability of the Pfaffian form $\delta Q_{\text {rev }}$, representing the infinitesimal heat exchanged reversibly [50-53,55-68]. In particular, we assume that the so-called metrical entropy $\mathcal{S}$ and absolute temperature $\mathcal{T}$ exist. Therefore, we can write

$\delta Q_{\mathrm{rev}}=\mathcal{T} \mathrm{d} \mathcal{S}$,

where $\mathcal{T} \geq 0$ is an integrating factor which satisfies

$\frac{\partial \mathcal{S}}{\partial \mathcal{M}} \equiv \frac{1}{\mathcal{T}}>0$,

so that

$\mathcal{T}(\mathcal{M}, \mathcal{J})=\frac{\left(\mathcal{M}+\sqrt{\mathcal{M}^{2}-\mathcal{J}^{2}}\right)^{2}-\mathcal{J}^{2}}{\left(\mathcal{M}+\sqrt{\mathcal{M}^{2}-\mathcal{J}^{2}}\right)^{3 / 2}}$.

In fact, if we choose the pair $(\mathcal{M}, \mathcal{J})$ as the extensive, independent variables in the equilibrium thermodynamics (i.e. homogeneous functions of degree one), then the homogeneity of the system is reflected in the integrability of the Pfaffian form

$\delta Q_{\text {rev }}=\mathrm{d} \mathcal{M}-\mathcal{W} \mathrm{d} \mathcal{J}$,

where $\mathcal{W}$ is the angular velocity of the black hole, given by

$\mathcal{W}(\mathcal{M}, \mathcal{J})=\frac{\mathcal{J}}{\mathcal{M}+\sqrt{\mathcal{M}^{2}-\mathcal{J}^{2}}}$.

Therefore, it is straightforward to show that, under the scaling transformation $(\mathcal{M}, \mathcal{J}) \mapsto(\lambda \mathcal{M}, \lambda \mathcal{J})$, we get $\delta Q_{\text {rev }} \mapsto$ $\lambda \delta Q_{\text {rev }}$, which means that the Pfaffian form is homogeneous of degree one. Consequently, we have an Euler vectorial field, or a Liouville operator, as the infinitesimal generator of the homogeneous transformations

$D=\mathcal{M} \frac{\partial}{\partial \mathcal{M}}+\mathcal{J} \frac{\partial}{\partial \mathcal{J}}$,

using which we obtain

$D \mathcal{S}=\frac{1}{2} \mathcal{S}$,

meaning that $\mathcal{S}$ is homogeneous of degree $1 / 2$. Similarly, the temperature is also homogeneous of degree $1 / 2$. Furthermore, it is straightforward to check that the angular velocity is a homogeneous function of degree zero, or $D \mathcal{W}=0$, and therefore, it is an intensive variable. 
It is naturally tempting to address a comparison with the natural $(3+1)$-dimensional counterpart (i.e. the Kerr-(Anti)de Sitter black hole). In fact, there are some differences between these cases that should be analyzed carefully. Furthermore, we have found a mathematical equivalence of a remarkable theoretical potential. However, keeping within the scope of this study, for now, we strive to present some immediate results of the above discussed concepts, and leave the aforementioned mathematical comparison to future work.

\section{The adiabatic-isoperimetral transformations}

An important result of the above approach is that it allows for the generation of a non-extremal manifold foliation. In fact, the non-extremal thermodynamic space is foliated by those submanifolds of co-dimension one, which are solutions of the Pfaffian equation $\delta Q_{\mathrm{rev}}=0$ [51].

As stated above, for the non-extremal manifold $(\mathcal{T}>0)$, the Pfaffian form is given by Eq. (12). Accordingly, performing the changes of variable $x=\mathcal{M}^{2}$ and $y=\mathcal{J}^{2}$, we get

$\delta Q_{\mathrm{rev}}=\frac{1}{2 \sqrt{x}} \mathrm{~d} x-\frac{1}{2(\sqrt{x}+\sqrt{x-y})} \mathrm{d} y$,

which respects the condition $x \geq y$. Thus, for the isoperimetral transformation $\delta Q_{\mathrm{rev}}=0$, which connects, adiabatically, the initial state $\mathbf{i} \equiv\left(x_{i}, y_{i}\right)$ to the final state $\mathbf{f} \equiv\left(x_{f}, y_{f}\right)$, the adiabatic trajectories are solutions to the Cauchy problem

$\frac{\mathrm{d} y}{\mathrm{~d} x}=1+\sqrt{1-\frac{y}{x}}$,

$y\left(x_{i}\right)=y_{i}$,

with $y_{i}<x_{i}$. It is then straightforward to show that the solutions to this problem are

$y_{a}(x)=2 \sqrt{\zeta_{a}} \sqrt{x}-\zeta_{a}$,

$y_{b}(x)=2 \sqrt{\zeta_{b}} \sqrt{x}-\zeta_{b}$,

where the constants $\zeta_{a, b} \equiv \zeta_{a, b}\left(x_{i}, y_{i}\right)$ are given by

$\zeta_{a, b}=2 x_{i}-y_{i} \pm 2 \sqrt{x_{i}\left(x_{i}-y_{i}\right)}$,

with $x_{i}>y_{i}$.

Each function vanishes at the point $\left(x_{0}, 0\right)$, with $x_{0}=$ $\zeta_{a, b} / 4$, which corresponds to the static BTZ black hole. The thermodynamic (extremal) limit, on the other hand, is reached at $\left(x_{e}, x_{e}\right)$, with $x_{e}=\zeta_{a, b}$ (see Fig. 1, showing the two functions intersecting at the initial point i). We will come back to these concepts later in this section.

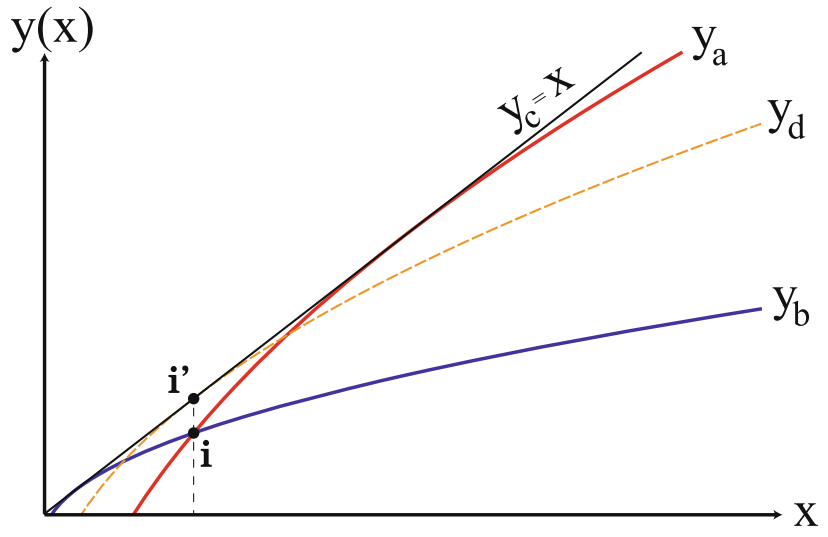

Fig. 1 The plots of the adiabatic solutions to the Cauchy problem, given in Eqs. (18a), (18b), (21a) and (21b). The static black hole limit, for each case, is where the curves hit the $x$ coordinate, whereas the extremal limit corresponds to the line $y=x$

Note that it is important to be cautious about the conditions on the extremal submanifold, on which the condition $\delta Q_{\text {rev }}=0$ is still satisfied. This implies that the extremal submanifold is an integral submanifold of the Pfaffian form $[51,53]$. In fact, considering the extremal point $\mathbf{i}^{\prime} \equiv\left(x_{i}, x_{i}\right)$ as initial state, the Cauchy problem becomes

$\frac{\mathrm{d} y}{\mathrm{~d} x}=1+\sqrt{1-\frac{y}{x}}$,

$y\left(x_{i}\right)=x_{i}$,

which allows for the two solutions

$y_{c}(x)=x$,

$y_{d}(x)=2 \sqrt{x_{i}} \sqrt{x}-x_{i}$.

Now that the solutions to both the non-extremal and extremal cases have been given, it is of importance to discuss their physical features, regarding the adiabatic processes. In particular, the solution $y_{a}$ given by Eq. (21a) indicates that the extremal states are adiabatically connected to each other. However, the solution $y_{b}$ in Eq. (21b) presents a more complicated situation, because it connects, adiabatically, the nonextremal states with the extremal ones. This, in fact, poses a contradiction to the second law of thermodynamics, since it provides the possibility to construct a Carnot cycle with one hundred percent thermal efficiency, and this violates Ostwald's postulate of the second law. Furthermore, it would be possible to transform completely the heat into work, which is also in contrast with the second law. To eliminate this singular behavior of the thermodynamic foliation, we assume that the surface $\mathcal{T}=0$ is a leaf itself, that is, we exclude it from the set of solutions. Accordingly, by introducing a discontinuity in $\mathcal{S}$ between the extremal and non-extremal states, we construct a foliation of the thermodynamic variety, whose 
leaves are distinguished by

$\mathcal{S}(\mathcal{M}, \mathcal{J})= \begin{cases}\mathcal{P} / 4, & \text { non-extremal states } \\ 0, & \text { extremal states. }\end{cases}$

The choice of the value $\mathcal{S}=0$ for the extremal states stems from some topological preferences $[69,70]$ and has been explicitly proposed by Carroll in Ref. [71]. Nevertheless, it has been shown that this choice is a particular case of a well-behaved area-dependent function, which can opt for non-zero values [72]. In particular, the thin shells (rings) in $(2+1)$-dimensional gravity, can change their entropy values during their evolution to a black hole (see Refs. [73-75]).

We can now establish the criteria for the physically acceptable solutions, based on the results obtained above:

1. Due to the homogeneity of the extensive variables $(\mathcal{M}, \mathcal{J})$ or $(x, y)$, every adiabatic process must satisfy

$$
\frac{\mathrm{d} y}{\mathrm{~d} x}>0
$$

2. An initial state belonging to the submanifold $\mathcal{T}>0$ can only be adiabatically connected to another state, if it neither belongs to the submanifold $\mathcal{T}=0$ nor passes through it.

3. In the neighborhood of any equilibrium state of the system, there exist states that are inaccessible by the reversible adiabatic processes (Carathéodory postulate) $[50,55,56]$.

Condition 1 is nothing but the result of expressing the thermodynamic system in terms of the extensive variables, which are, of course, homogeneous of degree one. Condition 2 ensures satisfaction of the second and third laws of thermodynamics. From the geometric point of view, this guarantees that the black hole topology does not change. The above statements have been visualized, qualitatively, in Fig. 2. There, we have exemplified the allowed processes by o $\leftrightarrow \mathrm{p}, \mathrm{r} \leftrightarrow \mathrm{s}$, and $\mathrm{q} \leftrightarrow \mathrm{t}$, and the forbidden processes by $\mathrm{p} \leftrightarrow \mathrm{q}$, and $\mathrm{q} \leftrightarrow$ r. Accordingly, the non-extremal initial state $\mathbf{i}$ is connected with the final states, by the adiabatic solution curves

$y(x)= \begin{cases}y_{a}(x), & \text { for } x_{0} \leq x<x_{i} \\ y_{b}(x), & \text { for } x_{i} \leq x<\infty\end{cases}$

In this sense, we can ramify the physically allowed branches of the solutions, as shown in Fig. 3. In this diagram, the physically accepted parts of the solution $y(x)$ are those that connect, adiabatically, the initial state $\mathbf{i} \equiv\left(x_{i}, y_{i}\right)$, with $y_{i}<$ $x_{i}$, to another state $\mathbf{f} \equiv\left(x_{f}, y_{f}\right)$, with $y_{f}<x_{f}$, following the $y_{a}$ branch, if $x_{f}<x_{i}$, and the $y_{b}$ branch, if $x_{f}>x_{i}$.

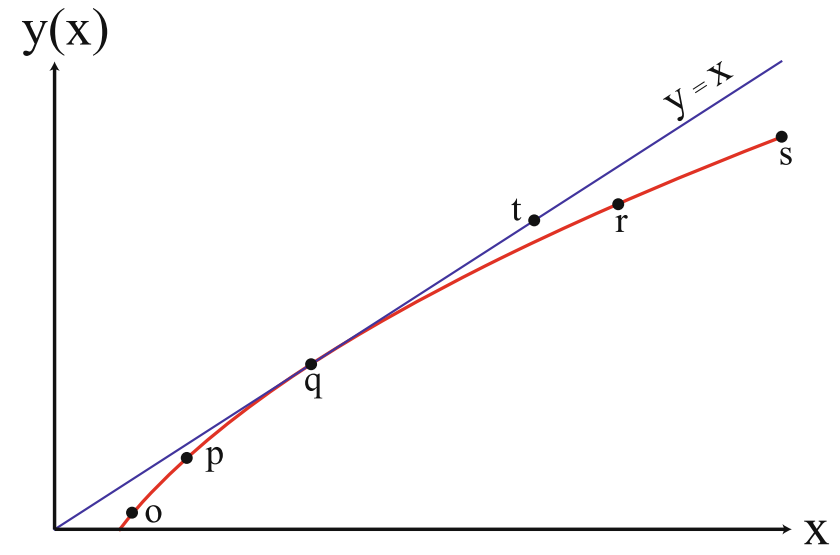

Fig. 2 The adiabatic solutions to the Cauchy problem and the extremal limit. In order to avoid violation of the second law, the only allowed processes are $\mathrm{o} \leftrightarrow \mathrm{p} ; \mathrm{r} \leftrightarrow \mathrm{s} ; \mathrm{q} \leftrightarrow \mathrm{t}$. The processes $\mathrm{p} \leftrightarrow \mathrm{q} ; \mathrm{q} \leftrightarrow \mathrm{r}$ are, on the other hand, prohibited

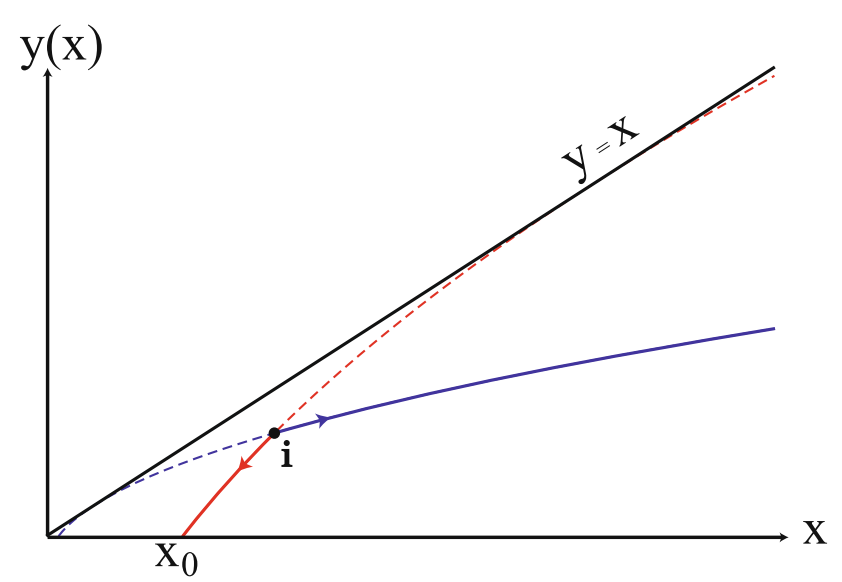

Fig. 3 The physically allowed solutions to the Cauchy problem for the BTZ black hole. The initial state $\mathbf{i} \equiv\left(x_{i}, y_{i}\right)$, with $y_{i}<x_{i}$, can be connected adiabatically to the final state $\mathbf{f} \equiv\left(x_{f}, y_{f}\right)$, with $y_{f}<x_{f}$, following the path $y_{a}$ (red curve) if $x_{f}<x_{i}$, and the path $y_{b}$ (blue curve) if $x_{f}>x_{i}$

A direct consequence of the condition 3 is that it prevents from forming an adiabatic cycle (as desired by engineering). Such a cycle is illustrated in Fig. 4. Referring to the triangular path in this diagram, the state $\mathbf{i}$, residing in the submanifold $\mathcal{T}>0$, is first connected adiabatically to the state $\mathbf{i}^{\prime}$, and then to the state $\mathbf{i}^{\prime \prime}$, which both reside in the submanifold $\mathcal{T}=0$, and finally, it is returned to $\mathbf{i}$. Note that the process $\mathbf{i}^{\prime} \rightarrow \mathbf{i}^{\prime \prime}$ is adiabatic and isothermal. In fact, since these states are inaccessible by the Carathéodory's postulate, the above adiabatic cycle is not allowed to form.

Accordingly, the extremal limit should be excluded from the adiabatic hypersurface, since there is no adiabatic process that can reach this state. In fact, either of the solutions (18a) and (18b) can produce an adiabatic surface that lies between the extremal $(y=x)$ and the static $(y=0)$ black hole limits (see Fig. 5). 


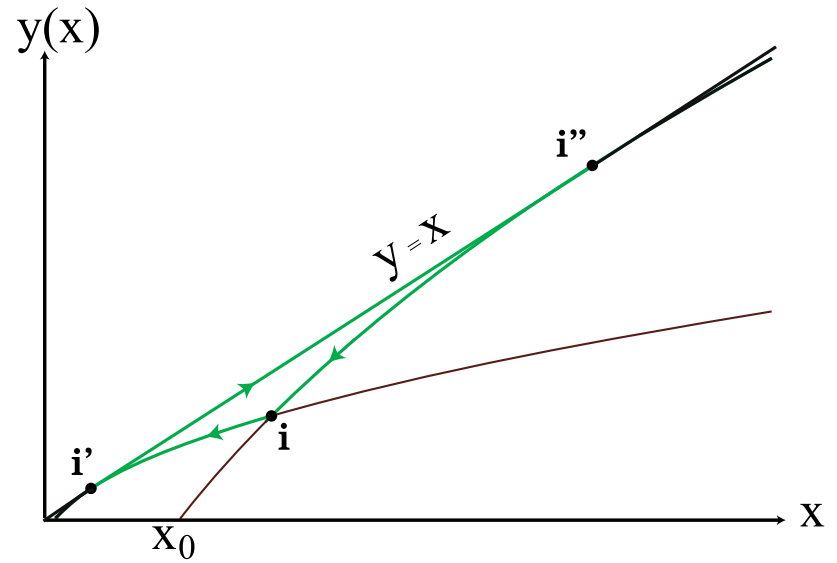

Fig. 4 The Carathéodory's postulate prevents the formation of an adiabatic cycle. In such a cycle, the equilibrium state $\mathbf{i}$ is adiabatically connected, respectively, to the extremal states $\mathbf{i}^{\prime}$ and $\mathbf{i}^{\prime \prime}$, and then is returned to itself (see the green triangular path). Such a cycle is prohibited, because the states $\mathbf{i}^{\prime}$ and $\mathbf{i}^{\prime \prime}$ are inaccessible for the state $\mathbf{i}$

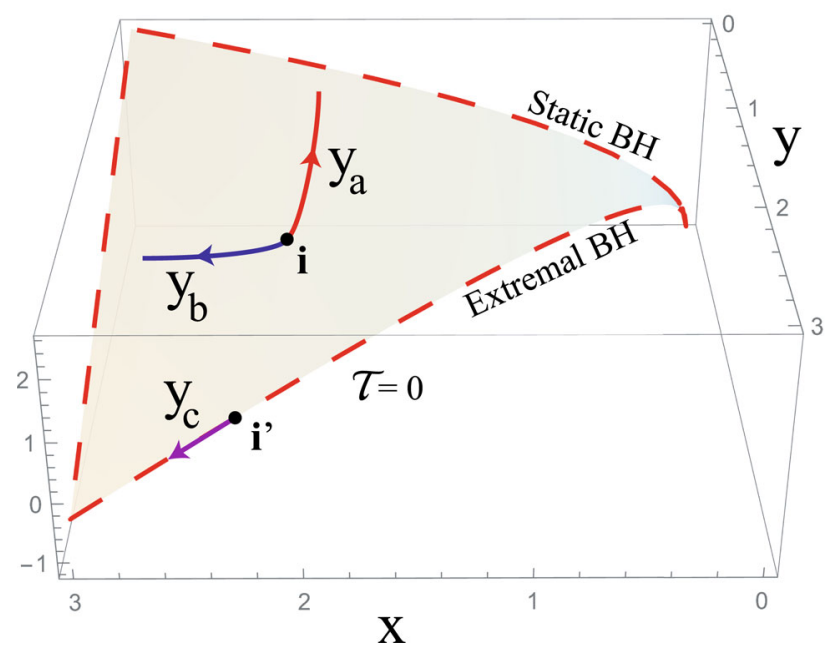

Fig. 5 The adiabatic surface plotted for $\zeta_{a}=1.2$, which is confined by the extremal, and the static black hole (BH) limits. The adiabatic processes $y_{a}$ and $y_{b}$, can connect the initial state $\mathbf{i}$ to other points on the surface. The same holds for the adiabatic process $y_{c}$ on the extremal limit, connecting the initial point $\mathbf{i}^{\prime}$ to other points on the same line. The transmission $\mathbf{i} \rightarrow \mathbf{i}^{\prime}$ is, however, prohibited by the Carathéodory's postulate

Note that, since the conditional solution given by Eq. (24) excludes the extremal states (the $\mathcal{T}=0$ leaf), we can ensure the satisfaction of the third law through all isoperimetral (or adiabatic) processes.

\section{Scattering of two extremal black holes and the second law}

In this section, we explore the possibility of an isolated merger occurring of two extremal BTZ black holes with the initial states $\left(\mathcal{M}_{1}, \mathcal{J}_{1}\right)$ and $\left(\mathcal{M}_{2}, \mathcal{J}_{2}\right)$, to produce the final state $\left(\mathcal{M}_{1}+\mathcal{M}_{2}, \mathcal{J}_{1}+\mathcal{J}_{2}\right)$. As in Refs. [53,76,77], we define the quantity

$\alpha^{2}(\mathcal{M}, \mathcal{J})=\mathcal{M}^{2}-\mathcal{J}^{2}$,

so that for the initial black holes we have $\alpha_{\text {in }}=\alpha_{1}+\alpha_{2}$, where

$\alpha_{1}^{2}\left(\mathcal{M}_{1}, \mathcal{J}_{1}\right)=\mathcal{M}_{1}^{2}-\mathcal{J}_{1}^{2} \geq 0$,

$\alpha_{2}^{2}\left(\mathcal{M}_{2}, \mathcal{J}_{2}\right)=\mathcal{M}_{2}^{2}-\mathcal{J}_{2}^{2} \geq 0$,

and for the final black hole, $\alpha_{\text {fin }}=\alpha_{12}$, with

$$
\begin{aligned}
& \alpha_{12}^{2}\left(\mathcal{M}_{1}+\mathcal{M}_{2}, \mathcal{J}_{1}+\mathcal{J}_{2}\right)=\left(\mathcal{M}_{1}+\mathcal{M}_{2}\right)^{2}-\left(\mathcal{J}_{1}+\mathcal{J}_{2}\right)^{2} \\
& =\alpha_{1}^{2}\left(\mathcal{M}_{1}, \mathcal{J}_{1}\right)+\alpha_{1}^{2}\left(\mathcal{M}_{2}, \mathcal{J}_{2}\right)+2\left(\mathcal{M}_{1} \mathcal{M}_{2}-\mathcal{J}_{1} \mathcal{J}_{2}\right)
\end{aligned}
$$

Therefore, for two extremal black holes of the initial states $\alpha_{1}^{2}\left(\mathcal{M}_{\text {ext }}^{(1)}, \mathcal{J}_{\text {ext }}^{(1)}\right)=\alpha_{2}^{2}\left(\mathcal{M}_{\text {ext }}^{(2)}, \mathcal{J}_{\text {ext }}^{(2)}\right)=0$, and for the positive masses $\mathcal{M}_{\text {ext }}=\left|\mathcal{J}_{\text {ext }}\right|>0$, Eq. (27) becomes

$$
\begin{aligned}
\alpha_{12}^{2} & \left(\mathcal{M}_{\mathrm{ext}}^{(1)}+\mathcal{M}_{\mathrm{ext}}^{(2)}, \mathcal{J}_{\mathrm{ext}}^{(1)}+\mathcal{J}_{\mathrm{ext}}^{(2)}\right) \\
= & 2\left(\mathcal{M}_{\mathrm{ext}}^{(1)} \mathcal{M}_{\mathrm{ext}}^{(2)}-\mathcal{J}_{\mathrm{ext}}^{(1)} \mathcal{J}_{\mathrm{ext}}^{(2)}\right) \\
= & 2\left(\left|\mathcal{J}_{\mathrm{ext}}^{(1)}\right|\left|\mathcal{J}_{\mathrm{ext}}^{(2)}\right|-\mathcal{J}_{\mathrm{ext}}^{(1)} \mathcal{J}_{\mathrm{ext}}^{(2)}\right) \\
= & 2\left|\mathcal{J}_{\mathrm{ext}}^{(1)}\right|\left|\mathcal{J}_{\mathrm{ext}}^{(2)}\right|(1-\cos \beta),
\end{aligned}
$$

where $\beta=\measuredangle\left(\mathcal{J}_{\text {ext }}^{(1)}, \mathcal{J}_{\text {ext }}^{(2)}\right)$. Thus, for extremal black holes rotating in the same direction $(\beta=0)$, the final black hole will be, as well, an extremal one. This is because, for those rotating in opposite directions $(\beta=\pi)$, the final black hole will not be extremal.

In the case of an extremal final state, a possible violation of the second law may occur, since, in fact, the process is irreversible and the entropy of the final state should be greater than that of the initial states (under the assumption that the system is isolated and there is no exchange of energy with the rest of the universe). On the other hand, if the final state is nonextremal, then its entropy is, naturally, greater than that of the initial states. As given in Eq. (22), the entropy of the BTZ black hole is $\mathcal{S}=g \mathcal{P} / 4$, where $\mathcal{P}$ is the perimeter of the event horizon and $g$ is the genus, which characterizes the topology of the thermodynamic manifold. In this sense, the extremal and non-extremal black holes are characterized, respectively, by $g=0$ and $g=1$. Hence, the latter corresponds to the change of the topology of the black hole.

In fact, passing from one black hole topology to another, we encounter the spacetime singularities. And since the above processes occur in the classical environment, these singularities are inevitable. Therefore, to avoid the complexities associated with the change in topology, it is convenient 
to infer that the scattering of two initially extremal BTZ black holes leads to an extremal BTZ black hole, and this violates the second law. The above statements can be summarized as

$\beta=\left\{\begin{array}{l}0 \Rightarrow \text { violation of the second law, } \pi \\ \Rightarrow \text { change of the black hole topology }\end{array}\right.$

\section{Discussion and final remarks}

In this paper we studied the thermodynamics of the BTZ black hole, based on the axiomatic approach of Carathéodory. We introduced the Pfaffian form $\delta Q_{\text {rev }}$ to represent the infinitesimal heat exchanged reversibly, which gives a definition to the metric entropy and temperature, with the latter as an integrating factor for the Pfaffian form. The natural extensive variables of the uncharged BTZ black hole in the equilibrium thermodynamics space (i.e. the homogeneous variables of degree one) are $(\mathcal{M}, \mathcal{J})$, which has an associated Pfaffian form $\delta Q_{\text {rev }}=\mathrm{d} \mathcal{M}-\mathcal{W} \mathrm{d} \mathcal{J}$. The symmetry of the homogeneity for $\delta Q_{\text {rev }}$ can then be inspected by means of the Euler vector field (Liouville operator) in Eq. (14), which indicates the consistency of the methods given here with the thermodynamic definition of the temperature.

As a first application of the approach presented, we studied adiabatic processes, by analyzing the corresponding Cauchy problem. In this sense, the problem is equivalent to the adiabatic processes in the Reissner-Nordström (RN) black hole spacetime [53], since, regarding the adiabatic transformations, the electric charge for the RN black hole plays the same role as the angular momentum for the BTZ black hole. We will address this issue in future work.

Since the obtained adiabatic solutions allow for two definite constants, they can be therefore employed in the correct physical description of the acceptable adiabatic paths. This way, and to respect the second, and especially the third law, the extremal submanifold $(\mathcal{T}=0)$ must be disconnected from the non-extremal one $(\mathcal{T}>0)$. In fact, the thermodynamic foliation of the non-extremal states allows us to have a consistent construction of thermodynamics, since there are proven arguments to connect the approaches of Carathéodory and Gibbs [65]. Consequently, the HawkingBekenstein entropy formula is found to be valid only for the non-extremal states. The entropy of the extremal states is, on the other hand, considered to be zero.

The classical merging of two extremal rotating BTZ black holes provided us another tool to inspect the leaf $\mathcal{T}=0$ and the corresponding property $\mathcal{S}=0$. The unconformity of the second law with the aforementioned entropy condition necessitates the inclusion of a net electrical charge for the black hole. In this sense, a new equivalence with the RN black hole could be found, which in that case relaxes the problem by introducing a definite angular momentum to the system [53].

Acknowledgements M. Fathi has been supported by the Agencia Nacional de Investigación y Desarrollo (ANID) through DOCTORADO Grants No. 2019-21190382, and No. 2021-242210002. J.V. was partially supported by the Centro de Astrofísica de Valparaíso (CAV).

Data Availability Statement This manuscript has no associated data or the data will not be deposited. [Authors' comment: This is a theoretical work and has no associated data.]

Open Access This article is licensed under a Creative Commons Attribution 4.0 International License, which permits use, sharing, adaptation, distribution and reproduction in any medium or format, as long as you give appropriate credit to the original author(s) and the source, provide a link to the Creative Commons licence, and indicate if changes were made. The images or other third party material in this article are included in the article's Creative Commons licence, unless indicated otherwise in a credit line to the material. If material is not included in the article's Creative Commons licence and your intended use is not permitted by statutory regulation or exceeds the permitted use, you will need to obtain permission directly from the copyright holder. To view a copy of this licence, visit http://creativecomm ons.org/licenses/by/4.0/.

Funded by SCOAP ${ }^{3}$.

\section{References}

1. A. Achúcarro, P. Townsend, A Chern-Simons action for threedimensional anti-de Sitter supergravity theories. Phys. Lett. B 180(1-2), 89-92 (1986)

2. E. Witten, $2+1$ dimensional gravity as an exactly soluble system. Nucl. Phys. B 311(1), 46-78 (1988)

3. M. Bañados, C. Teitelboim, J. Zanelli, The black hole in threedimensional space-time. Phys. Rev. Lett. 69, 1849-1851 (1992)

4. M. Bañados, M. Henneaux, C. Teitelboim, J. Zanelli, Geometry of the (2+1) black hole. Phys. Rev. D 48, 1506-1525 (1993) [Erratum: Phys. Rev. D 88, 069902 (2013)]

5. S. Carlip, The (2+1)-Dimensional black hole. Class. Quantum Gravity 12, 2853-2880 (1995)

6. M. Bañados, Three-dimensional quantum geometry and black holes. AIP Conf. Proc. 484(1), 147-169 (1999)

7. M. Cataldo, S. del Campo, A.A. García, BTZ black hole from (3+1) gravity. Gen. Relativ. Gravit. 33, 1245-1255 (2001)

8. E. Ayon-Beato, C. Martinez, J. Zanelli, Birkhoff's theorem for three-dimensional AdS gravity. Phys. Rev. D 70, 044027 (2004)

9. E. Witten, Three-dimensional gravity revisited, arXiv e-prints, arXiv:0706.3359 (2007)

10. N. Cruz, C. Martínez, L. Peña, Geodesic structure of the $(2+1)$ dimensional BTZ black hole. Class. Quantum Gravity 11(11), 2731-2739 (1994)

11. J. Gamboa, F. Méndez, Scattering in three dimensional extremal black holes. Class. Quantum Gravity 18, 225-232 (2001)

12. S. Lepe, F. Méndez, J. Saavedra, L. Vergara, Fermions scattering in a three-dimensional extreme black hole background. Class. Quantum Gravity 20, 2417-2428 (2003)

13. V. Cardoso, J.P.S. Lemos, Scalar, electromagnetic and Weyl perturbations of BTZ black holes: quasinormal modes. Phys. Rev. D 63, 124015 (2001)

14. D. Birmingham, S. Carlip, Y.-J. Chen, Quasinormal modes and black hole quantum mechanics in (2+1)-dimensions. Class. Quantum Gravity 20, L239-L244 (2003) 
15. J. Crisostomo, S. Lepe, J. Saavedra, Quasinormal modes of extremal BTZ black hole. Class. Quantum Gravity 21, 2801-2810 (2004)

16. M.R. Setare, Nonrotating BTZ black hole area spectrum from quasinormal modes. Class. Quantum Gravity 21, 1453-1458 (2004)

17. N. Cruz, J. Zanelli, Stellar equilibrium in (2+1)-dimensions. Class. Quantum Gravity 12, 975-982 (1995)

18. A.A. García, Stationary circularly symmetric $2+1$ rigidly rotating perfect fluids. Phys. Rev. D 69, 124024 (2004)

19. A.A. García, C. Campuzano, All static circularly symmetric perfect fluid solutions of (2+1) gravity. Phys. Rev. D 67, 064014 (2003)

20. N. Cruz, M. Olivares, J.R. Villanueva, Static circularly symmetric perfect fluid solutions with an exterior BTZ metric. Gen. Relativ. Gravit. 37, 667-674 (2005)

21. C. Gundlach, P. Bourg, Rigidly rotating perfect fluid stars in $2+1$ dimensions. Phys. Rev. D 102(8), 084023 (2020)

22. A. Rincón, B. Koch, Scale-dependent BTZ black hole. Eur. Phys. J. C 78(12), 1022 (2018)

23. A. Rincón, E. Contreras, P. Bargueño, B. Koch, G. Panotopoulos, Scale-dependent $(2+1)$-dimensional electrically charged black holes in Einstein-power-Maxwell theory. Eur. Phys. J. C 78(8), 641 (2018)

24. A. Rincón, J.R. Villanueva, The Sagnac effect on a scale-dependent rotating BTZ black hole background. Class. Quantum Gravity 37(17), 175003 (2020)

25. M. Fathi, A. Rincón, J.R. Villanueva, Photon trajectories on a first order scale-dependent static BTZ black hole. Class. Quantum Gravity 37(7), 075004 (2020)

26. A. Rincón, E. Contreras, F. Tello-Ortíz, P. Bargueño, G. Abellán, Anisotropic $2+1$ dimensional black holes by gravitational decoupling. Eur. Phys. J. C 80(6), 490 (2020)

27. E.C. Vagenas, Energy distribution in a BTZ black hole spacetime. Int. J. Mod. Phys. D 14, 573-586 (2005)

28. M. Cataldo, A.A. García, Regular (2+1)-dimensional black holes within nonlinear electrodynamics. Phys. Rev. D 61, 084003 (2000)

29. M. Cataldo, P. Salgado, Three dimensional extreme black hole with self (anti-self) dual Maxwell field. Phys. Lett. B 448, 20-25 (1999)

30. S. Carlip, Conformal field theory, (2+1)-dimensional gravity, and the BTZ black hole. Class. Quantum Gravity 22, R85-R124 (2005)

31. R.G. Cai, Z.J. Lu, Y.Z. Zhang, Critical behavior in $(2+1)-$ dimensional black holes. Phys. Rev. D 55, 853-860 (1997)

32. M. Bañados, T. Brotz, M.E. Ortíz, Boundary dynamics and the statistical mechanics of the (2+1)-dimensional black hole. Nucl. Phys. B 545, 340-370 (1999)

33. S. Wang, S.-Q. Wu, F. Xie, L. Dan, The First laws of thermodynamics of the (2+1)-dimensional BTZ black holes and Kerr-de Sitter spacetimes. Chin. Phys. Lett. 23, 1096-1098 (2006)

34. B.P. Dolan, The cosmological constant and the black hole equation of state. Class. Quantum Gravity 28, 125020 (2011)

35. T. Sarkar, G. Sengupta, B.N. Tiwari, On the thermodynamic geometry of BTZ black holes. J. High Energy Phys. 2006(11), 015-015 (2006)

36. H. Quevedo, A. Sanchez, Geometric description of BTZ black holes thermodynamics. Phys. Rev. D 79, 024012 (2009)

37. M. Akbar, H. Quevedo, K. Saifullah, A. Sanchez, S. Taj, Thermodynamic geometry of charged rotating BTZ lack holes. Phys. Rev. D 83, 084031 (2011)

38. S.H. Hendi, S. Panahiyan, B.E. Panah, M. Momennia, A new approach toward geometrical concept of black hole thermodynamics. Eur. Phys. J. C 75, 507 (2015)

39. S.H. Hendi, B.E. Panah, S. Panahiyan, Massive charged BTZ black holes in asymptotically (a)dS spacetimes. J. High Energy Phys. 2016, 29 (2016)

40. D.V. Singh, S. Siwach, Thermodynamics of BTZ black hole and entanglement entropy. J. Phys. Conf. Ser. 481, 012014 (2014)
41. S. Alsaleh, Thermodynamics of BTZ black holes in gravity's rainbow. Int. J. Mod. Phys. A 32(15), 1750076 (2017)

42. M. Dehghani, Thermodynamics of charged dilatonic btz black holes in rainbow gravity. Phys. Lett. B 777, 351-360 (2018)

43. T. Liang, W. Tang, W. Xu, Entropy relations and bounds of BTZ black hole in gravity's rainbow. Int. J. Mod. Phys. D 28(08), 1950109 (2019)

44. U. Camci, Three-dimensional black holes via Noether symmetries. Phys. Rev. D 103(2), 024001 (2021)

45. S. Chougule, S. Dey, B. Pourhassan, M. Faizal, BTZ black holes in massive gravity. Eur. Phys. J. C 78(8), 685 (2018)

46. M. Bravo-Gaete, M. Hassaine, Thermodynamics of a BTZ black hole solution with an Horndeski source. Phys. Rev. D 90(2), 024008 (2014)

47. L. Ortíz, N. Bretón, Aspects of the BTZ black hole interacting with fields. Mod. Phys. Lett. A 34(31), 1950251 (2019)

48. P.K. Townsend, B. Zhang, Thermodynamics of "Exotic" BañadosTeitelboim-Zanelli black holes. Phys. Rev. Lett. 110(24), 241302 (2013)

49. V.V. Kiselev, Entropy of BTZ black hole and its spectrum by quantum radial geodesics behind horizons. Phys. Rev. D 73, 104018 (2006)

50. C. Carathéodory, Untersuchungen über die Grundlagen der Thermodynamik. Math. Ann. 67, 355-386 (1909)

51. F. Belgiorno, Black hole thermodynamics in Caratheodory's approach. Phys. Lett. A 312, 324-330 (2003)

52. F. Belgiorno, S.L. Cacciatori, General symmetries: from homogeneous thermodynamics to black holes. Eur. Phys. J. Plus 126, 86 (2011)

53. F. Belgiorno, M. Martellini, Black holes and the third law of thermodynamics. Int. J. Mod. Phys. D 13, 739-770 (2004)

54. N. Cruz, S. Lepe, On the thermal description of the BTZ black holes. Phys. Lett. B 593, 235-241 (2004)

55. H.A. Buchdahl, On the principle of carathéodory. Am. J. Phys. 17(1), 41-43 (1949)

56. H.A. Buchdahl, On the theorem of carathéodory. Am. J. Phys. 17(1), 44-46 (1949)

57. H.A. Buchdahl, On the unrestricted theorem of carathéodory and its application in the treatment of the second law of thermodynamics. Am. J. Phys. 17(4), 212-218 (1949)

58. H.A. Buchdahl, Integrability conditions and carathéodory's theorem. Am. J. Phys. 22(4), 182-183 (1954)

59. H.A. Buchdahl, Simplification of a proof of carathéodory's theorem. Am. J. Phys. 23(1), 65-66 (1955)

60. P.T. Landsberg, A deduction of carathéodory's principle from kelvin's principle. Nature 201, 485-486 (1964)

61. T.W. Marshall, A simplified version of Carathéodory thermodynamics. Am. J. Phys. 46(2), 136-137 (1978)

62. J. Boyling, Carathéodory's principle and the existence of global integrating factors. Commun. Math. Phys. 10, 52-68 (1968)

63. J. Boyling, An axiomatic approach to classical thermodynamics. Proc. R. Soc. Lond. A 329, 35-70 (1972)

64. L. Pogliani, M. Berberan-Santos, Constantin Carathéodory and the axiomatic thermodynamics. J. Math. Chem. 28, 313-324 (2000)

65. F. Belgiorno, Homogeneity as a bridge between Carathéodory and Gibbs, arXiv e-prints, arXiv:math-ph/0210011 (2002)

66. F. Belgiorno, Quasihomogeneous thermodynamics and black holes. J. Math. Phys. 44, 1089-1128 (2003)

67. F. Belgiorno, Notes on the third law of thermodynamics: I. J. Phys. A Math. Gen. 36(30), 8165-8193 (2003)

68. F. Belgiorno, Notes on the third law of thermodynamics: II. J. Phys. A Math. Gen. 36(30), 8195-8221 (2003)

69. S.W. Hawking, G.T. Horowitz, S.F. Ross, Entropy, area, and black hole pairs. Phys. Rev. D 51, 4302-4314 (1995) 
70. C. Teitelboim, Action and entropy of extreme and nonextreme black holes. Phys. Rev. D 51, 4315 (1995). [Erratum: Phys. Rev. D 52, 6201 (1995)]

71. S.M. Carroll, M.C. Johnson, L. Randall, Extremal limits and black hole entropy. J. High Energy Phys. 2009(11), 109-109 (2009)

72. J.P. Lemos, G.M. Quinta, O.B. Zaslavskii, Entropy of extremal black holes: horizon limits through charged thin shells in a unified approach. Phys. Rev. D 93(8), 084008 (2016)

73. J.P. Lemos, G.M. Quinta, Entropy of thin shells in a $(2+1)$ dimensional asymptotically AdS spacetime and the BTZ black hole limit. Phys. Rev. D 89(8), 084051 (2014)
74. J.P. Lemos, F.J. Lopes, M. Minamitsuji, J.V. Rocha, Thermodynamics of rotating thin shells in the BTZ spacetime. Phys. Rev. D 92(6), 064012 (2015)

75. J.P. Lemos, M. Minamitsuji, O.B. Zaslavskii, Unified approach to the entropy of an extremal rotating BTZ black hole: thin shells and horizon limits. Phys. Rev. D 96(8), 084068 (2017)

76. M. Molina, J.R. Villanueva, On the thermodynamics of the Hayward black hole. Class. Quantum Gravity 38(10), 105002 (2021)

77. M. Fathi, M. Molina, J.R. Villanueva, Adiabatic evolution of Hayward black hole, arXiv e-prints, arXiv:2101.12253 (2021) 